\title{
Viral encephalitis complicated by acute retinal necrosis syndrome: A case report
}

\author{
ZHI-GANG LIANG, ZHU-LI LIU, XU-WEN SUN, MAN-LI TAO and GUO-PING YU \\ Department of Neurology, Affiliated Yantai Yuhuangding Hospital of Qingdao University Medical College, \\ Yantai, Shandong 264000, P.R. China
}

Received January 11, 2014; Accepted November 10, 2014

DOI: $10.3892 /$ etm.2015.2557

\begin{abstract}
Acute retinal necrosis syndrome (ARN) is a viral infection characterized by focal retinal necrosis. Viral meningitis complicated by ARN is relatively rare. In the present case study, a 44-year-old male presented with fever, headache and mental disorder. After four days, the patient developed blurred vision. The patient was diagnosed with viral encephalitis complicated by bilateral ARN, based on the examination results. After treatment with antivirals and systemic glucocorticoids, the symptoms of the patient improved. Viral encephalitis may be an important risk factor for ARN. For a patient with viral encephalitis who experiences decreased visual acuity or vitreous opacification, the possibility of ARN should be considered.
\end{abstract}

\section{Introduction}

Viral encephalitis is an inflammation of the central nervous system caused by a specific virus. The prognosis is often good if treated early. Acute retinal necrosis syndrome (ARN) is a viral infection characterized by retinal focal necrosis (1). A previous study reported an incidence of 1 case of ARN per 1.6-2.0 million population (2). ARN is a viral inflammatory condition that manifests with vitreitis, severe retinal vasculitis and progressive peripheral retinal necrosis (3). Previous studies have shown that ARN may exist concurrently with viral encephalitis $(4,5)$. The present study describes the case of a patient with viral encephalitis complicated by bilateral ARN. Written informed consent was obtained from the patient. The case report and a review of the literature were subsequently utilized to investigate the possible pathogenesis of this disease.

Correspondence to: Dr Zhi-Gang Liang, Department of Neurology, Affiliated Yantai Yuhuangding Hospital of Qingdao University Medical College, 20 East Road of Yuhuangding, Yantai, Shandong 264000, P.R. China

E-mail: zgliang@hotmail.com

Key words: viral encephalitis, acute retinal necrosis, fundus fluorescein angiography, fundus laser

\section{Case report}

A 44-year-old male patient was admitted to the Department of Neurology in the Affiliated Yantai Yuhuangding Hospital of Qingdao University Medical College (Yantai, China) on June 2, 2012 with a fever and headache that had persisted for 12 days, and confusion and divagation that had lasted for 1 day. The patient had no remarkable clinical or family history and had no history of drug allergies. Physical examination revealed that the patient was restless, irritable, had neck stiffness and was positive for Kernig's sign. The patient's deep tendon reflexes were normal and no paresis was present. Blood chemistry and urinalysis tests were normal. The results from the cerebrospinal fluid (CSF) analysis were as follows: leucocytes, $805.3 \mathrm{mg} / 1$; monocytes (90\%), 130x10\% $/ 1$; and herpes simplex virus I (HSV-I) immunoglobulin M, positive. Polymerase chain reaction analysis revealed positive results for HSV in the CSF and blood, while tests for cytomegalovirus (CWV) allotype, human immunodeficiency virus and hepatitis $\mathrm{C}$ were negative. The culture of CSF produced no acid-fast bacilli, common bacteria or Cryptococci. Based on these results, the patient was diagnosed with viral encephalitis and administered antiviral therapy (500 $\mathrm{mg}$ acyclovir intravenously every $8 \mathrm{~h}$, dropwise).

Following antiviral therapy, the general condition of the patient improved; however, on June 6, 2012 he presented with blurred vision. Ophthalmological examinations revealed mild opacification of the lenses and moderate opacification of the vitreous body. The fundus appeared hazy and had blurred disc margins. Ophthalmic ultrasound showed a dense hypoechoic focal lesion (Fig. 1). The mean visual evoked potential amplitude was slightly decreased and the time-to-peak was substantially prolonged. Methylprednisolone pulse therapy $(1.0 \mathrm{~g} /$ day $)$ was administered for three days, followed by orally administered prednisone. A second ophthalmological examination (June 16,2012) revealed that vitreous opacification was present in both eyes. The majority of the retinal artery was occluded, particularly in the left eye. Retinal necrotic lesions with mild bleeding were visible.

The examination results confirmed the patient had viral encephalitis with bilateral ARN, and retinal laser treatment was administered. Two days after retinal laser treatment, visual acuity had improved. The CSF tests (June 18, 2012) were found to be normal. Fluorescein fundus angiography revealed that 


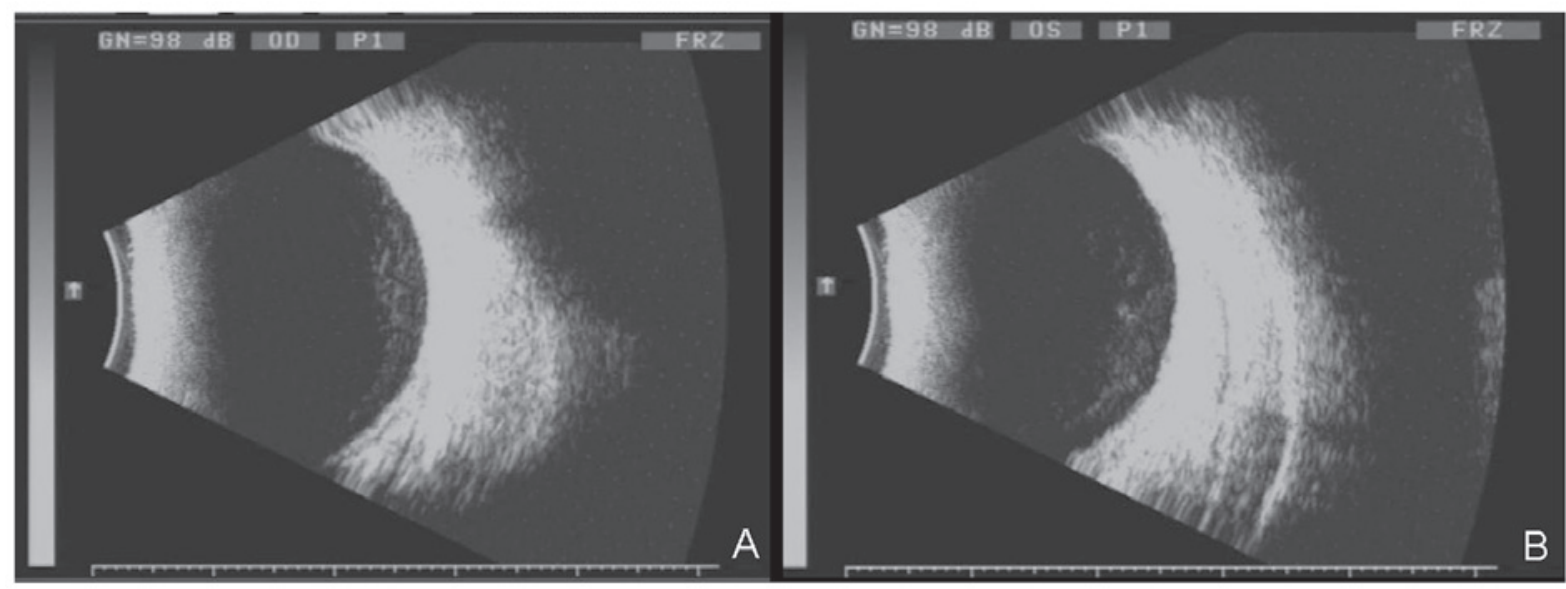

Figure 1. Ophthalmic ultrasound of the (A) right and (B) left eye. In both eyes, the fundus appeared hazy with blurred disc margins; no retinal edema was observed.

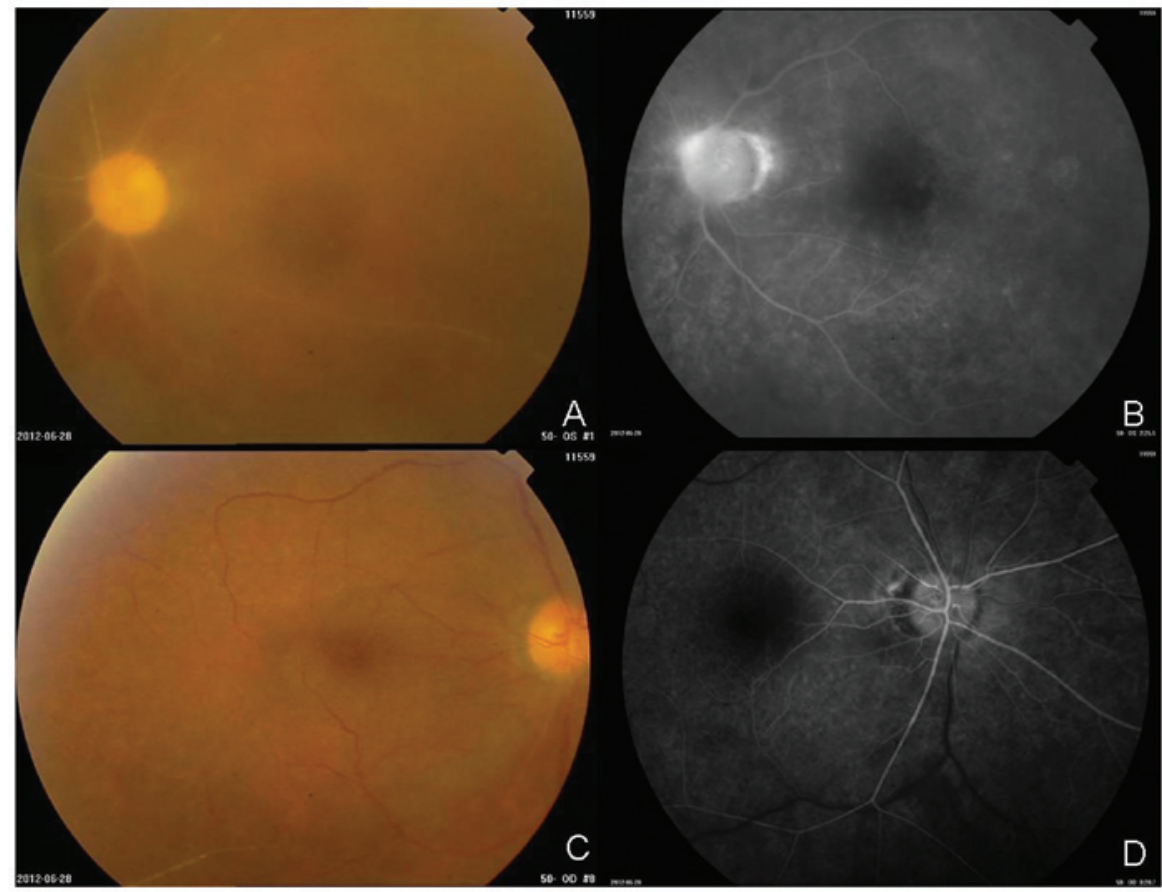

Figure 2. Fluorescein fundus angiography of the (A and B) right and (C and D) left fundus. (A and B) The enlarged retinal vessels in the medial side of macula lutea were not filled after one minute and dye perfusion was delayed. (C and D) The retinal artery was thin and unevenly distributed. Slight fluorescein leakage and pigmentation were visible on the artery wall. Fluorescein retinal angiography showed intense late fluorescence of the optic disc. With the exception of the large vessels surrounding the optic disc, no retinal vessels were filled in the left eye.

the majority of the retinal artery, shown as a white line, was occluded, particularly in the left eye (Fig. 2). Retinal necrotic lesions, together with mild bleeding, were visible along the white line. Visual acuity was markedly improved following treatment.

\section{Discussion}

Viral meningitis complicated by ARN is relatively rare, and is most common in immunocompromised individuals. Bilateral eye involvement occurs only in approximately one-third of patients with ARN (6). The present case study involved an adult male with viral encephalitis complicated by bilateral
ARN. The mechanism by which viruses induce ARN has yet to be elucidated; however, it has been suggested that the virus may be transmitted along the brain-optic nerve axon-retina pathway $(7,8)$.

Viral encephalitis may be an important risk factor for ARN. ARN syndrome is known to occur occasionally alongside, or shortly after, herpetic encephalitis (9). A review of the available literature (10), as well as our own observations, indicates that there is no universal guideline on how long antiviral and anti-inflammatory treatment should be continued. Early retinal laser treatment may prevent retinal detachment in patients with ARN (11). At present, a full course of antiviral therapy is essential to prevent the development of ARN as a result of viral 
encephalitis, as well as to prevent the progression of ARN from one eye to the other (12). Systemic glucocorticoids may alleviate retinal inflammation, and have also been shown to protect the retina and optic nerve. Prophylactic laser coagulation of retinal defects and margins may also prevent retinal detachment (13).

In conclusion, if a patient with viral encephalitis experiences decreased visual acuity or has vitreous opacification, the possibility of ARN should be considered. Fundus fluorescein angiography should be performed and early active treatment should be applied.

\section{References}

1. Kanoff J and Sobrin L: New diagnosis and treatment paradigms in acute retinal necrosis. Int Ophthalmol Clin 51: 25-31, 2011.

2. Muthiah MN, Michaelides M, Child CS and Mitchell SM: Acute retinal necrosis: a national population-based study to assess the incidence, methods of diagnosis, treatment strategies and outcomes in the UK. Br J Ophthalmol 91: 1452-1455, 2007.

3. Lau CH, Missotten T, Salzmann J and Lightman SL: Acute retinal necrosis features, management, and outcomes. Ophthalmology 114: 756-762, 2007.

4. Vandercam T, Hintzen RQ, de Boer JH and Van der Lelij A: Herpetic encephalitis is a risk factor for acute retinal necrosis. Neurology 71: 1268-1274, 2008.
5. Klein A and Lefebvre P: Three consecutive episodes of acute retinal necrosis due to herpes simplex-1 over twelve years following herpetic encephalitis. Ocul Immunol Inflamm 15: 411-413, 2007.

6. Gartry DS, Spalton DJ, Tilzey A and Hykin PG: Acute retinal necrosis syndrome. Br J Ophthalmol 75: 292-297, 1991.

7. Cardine S, Chaze PA, Bourcier F, et al: Bilateral acute retinal necrosis syndrome associated with meningoencephalitis caused by herpes simplex virus 2. A case report. J Fr Ophtalmol 27: 795-800, 2004 (In French).

8. Arruti M, Aldazabal M, Blanco A, et al: Acute herpes simplex virus type 1 retinal necrosis three years after herpes simplex encephalitis. Rev Neurol 58: 45-46, 2014 (In Spanish).

9. Gaynor BD, Wade NK and Cunningham ET Jr: Herpes simplex virus type 1 associated acute retinal necrosis following encephalitis. Retina 21: 688-690, 2001.

10. Flaxel CJ, Yeh S and Lauer AK: Combination systemic and intravitreal antiviral therapy in the management of acute retinal necrosis syndrome (an American Ophthamological Society thesis). Trans Am Ophthalmol Soc 111: 133-144, 2013.

11. Kianersi F, Masjedi A and Ghanbari H: Acute retinal necrosis after herpetic encephalitis. Case Rep Ophthalmol 1: 85-89, 2010.

12. Tibbetts MD, Shah CP, Young LH, et al: Treatment of acute retinal necrosis. Ophthalmology 117: 818-824, 2010.

13. Verma L, Venkatesh P, Satpal G, Rathore K and Tewari HK: Bilateral necrotizing herpetic retinopathy three years after herpes simplex encephalitis following pulse corticosteroid treatment. Retina 19: 464-467, 1999. 